\title{
A Study of the Electric Response of He II at the Excitation of Second Sound Waves
}

\author{
Tymofiy V. Chagovets \\ Institute of Physics ASCR, Na Slovance 2, 18221 Prague, \\ Czech Republic
}

July 28, 2021

\begin{abstract}
We report an experimental investigation of the electric response of superfluid helium. Our results confirm the presence of electric potential that appears at the relative oscillatory motion of normal fluid and superfluid components in helium generated by the heater. The resonance of the electric potential was observed in the first four harmonics. A suggested method for the detection of the electric response allows the required resonance peak to be distinguished from spurious signals. Our results are in qualitative agreement with the data published by previous researchers. The reasons for the discrepancy in the measured values of the potential difference are discussed.
\end{abstract}

Keywords: Superfluid ${ }^{4} \mathrm{He}$, Second sound, Polarization, Electric Potential

PACS 05.70Ln, 05.70Jk

\section{Introduction}

Superfluid helium or He II is a macroscopic quantum fluid that exhibits extraordinary properties. The behavior of the fluid can be understood using a Landau - Tisza model, where He II is considered to be a two-component fluid with independent velocity fields: the inviscid superfluid of density $\rho_{s}$, and the normal fluid of density $\rho_{n}$, where the total density $\rho=\rho_{s}+\rho_{n}$ [1, 2]. The superfluid has neither viscosity nor entropy, and the entire heat content of He II is carried by the normal component. This simplified picture is described by two fluid equations of motion. One of the important outcome of these equations is not only the existence of density fluctuations relative to ordinary or first sound, but also a prediction of the second sound, a wave described by temperature fluctuations.

Recent experiments [3, 4, reported another exceptional property of superfluid ${ }^{4} \mathrm{He}$, such as electrical activity that appears at the relative oscillatory motion of the normal fluid and superfluid components, or second sound wave. A standing half-wave of second sound was generated by a heater, which was placed on one end of a dielectric resonator. The AC potential difference between an electrode that was placed on the opposite end of the resonator and the ground was recorded. The resonance frequency of the electric 
response corresponded to the frequency of the second sound resonance. The experiments showed that the amplitude of the electric potential, $\Delta U$, is proportional to the amplitude of the temperature oscillations, $\Delta T$, in a second sound wave. The ratio $\Delta T / \Delta U \approx 2 e / k_{B}$ was independent of the resonator size and the temperature of the bath in the range 1.4$1.8 \mathrm{~K}$. Here, $e$ is the electron charge, and $k_{B}$ is the Boltzmann constant. It should be noted that experiments with the first sound in both He I and He II did not show any electrical signal, even when high power was applied to the sound emitter $[3]$. This electrical activity of superfluid helium is rather puzzling. The helium atom is not only chemically inert and electrically neutral and does not have an electric dipole moment, but it also has the highest ionization potential of any element and, correspondingly, an exceptionally low polarizability.

The experiments of Ref. 3, 4 stimulated a large number of theoretical studies and suggested various theoretical explanations for this phenomenon [5, 6, 7, 8, 9, 10, 11, 12. For instance, in Ref. [6], it was assumed that superfluid helium has an ordered electric quadrupole moment that can be polarized by the nonuniform flow of the superfluid. However, the microscopic nature of such a quadrupole moment was not discussed and remains an unanswered question. The author of Ref. [7] suggested the inertial mechanism of polarization of ${ }^{4} \mathrm{He}$ atoms based on the large mass difference between electrons and nuclei. An analog of this polarization occurs in metals and is called the Stewart - Tolman effect. However, this approach supposes the polarization of helium by first sound, which contradicts the experimental results of Ref. 3]. Moreover, this theoretical model predicts a temperature dependence for the $\Delta T / \Delta U$ ratio, which also contradicts the experimental results. In Ref. [5], the electrical activity was interpreted based on a similar inertial effect as in Ref. [7], which arises under the influence of a centrifugal force causing a nonuniform azimuthal rotational velocity of the superfluid component around the axis of a quantum vortex in He II. However, in this case, macroscopic polarization requires a relatively high concentration of vortex lines. Thus, the theoretical models are discussed with regard to the possible explanations and contradictions that arise in understanding the experimental data, but a satisfactory explanation of this phenomenon has not yet been found.

Additionally, detailed analysis of the articles [3, 4, revealed a number of questions that need to be addressed. For example, the amplitude of the signals is relatively small (approximately $100 \mathrm{nV}$ ), and measuring such signals can be difficult due to various sources of noise. In this report, we will demonstrate the existence of a large number of spurious peaks in a frequency sweep that have amplitudes comparable to that of the signal of interest. These spurious peaks can potentially be misinterpreted as the required electric response. In the original experiments, the authors used a measuring loop with the compensation of the input capacitance. Such a measuring circuit itself can be a source of electric pickup or spurious signals with amplitudes comparable to the observed electric response. Unfortunately, the original papers [3, 4] do not include some rather important information, such as the principal scheme of the measuring circuit, the capacitance of the connecting wires and electrodes used in the experiment, and the area of the resonator's cross-section. Moreover, the analysis of the experimental data shows some contradictions in experimental data reported in 3 and 4 that reduce confidence in these results and may give a most misleading impression. For example, $\Delta \mathrm{T}$ values, reported in [3] and [4, is supposed to be related to the same experiment, however they are approximately one order of magnitude higher. Therefore, the electric response in 
He II is require detailed experimental investigation with confirmation of some previous results.

The singularity of the phenomenon, the relatively small amplitude of the measured signal, and the lack of some important experimental details in the original papers motivated further study of the electric response in He II. The absence of new experimental data and of an appropriate theoretical model for the last 10 years makes this issue even more interesting. The purpose of this work is detailed verification of correlation between the second sound resonance and the resonance of electric response, test the existence of the electric response on higher modes of the second sound. The investigation this phenomenon is also requires a test various measuring circuits without compensation of the input capacitance and develop a reliable method of detecting the electric response signal in He II.

\section{$2 \quad$ Experimental setup}

Our experiments were performed in an open bath cryostat, and a pumping unit was used to maintain the temperature in the range 1.4-2.2 $\mathrm{K}$. The helium bath temperature was monitored and stabilized at the chosen value using a Conductus LTC-21 temperature controller with a calibrated Cernox sensor and a $50 \Omega$ heater mounted on the bottom of the cryostat. With this system, we were able to stabilize the bath at temperatures of up to $\pm 100 \mu \mathrm{K}$.

The resonator, which was $25-\mathrm{mm}$ long and had an inner diameter of $7 \mathrm{~mm}$, was machined from a piece of epoxy, and its inner surface was polished. Both ends of the resonator can be covered by flanges with various attached sensors, for example, a heater with bifilar wire or a thin-film thermometer, or mounted with electrodes. The resonator was hung on a support inside a metallic shielded box and had no electrical contact with the metallic parts of the cryostat. All sensors were connected to the measuring circuit with low temperature coax cables [13, and the total capacity of the loop was approximately $260 \mathrm{pF}$.

To excite the second sound wave, we used a manganin heater with approximately $180 \Omega$ resistance, which was glued with Varnish glue to the surface of a polished brass disk with a thickness of $4 \mathrm{~mm}$ that was connected to one end of the resonator. The heater, which had a spiral shape, was made of two layers bifilar wire that covered the entire cross-section of the channel.

The initial experiments [3, 4] showed low peak values of the resonance of approximately $10^{-7} \mathrm{~V}$ with a peak width of approximately $2 \mathrm{~Hz}$. Identifying such small signals is a rather complicated task due to the relatively high noise level, which was only 5 times lower than the magnitude of the signal. The low signal-to-noise ratio and small width of the resonance peak require measurements with a large time constant (usually 1 or 3 seconds) for the frequency sweep with a step of approximately $10 \mathrm{mHz}$. The increase in the number of points allows to receive a resonant curve of more regular shape. These types of measurement occur over a long period of time, and the identification of the resonance peak is difficult because of the wide range of frequencies. Nevertheless, the range of interest can be determined analytically.

As was discovered in Ref [3], the resonance frequency of the electric response coincides with the resonance frequency of the second sound. The resonance frequency, $f_{r}$, of second 


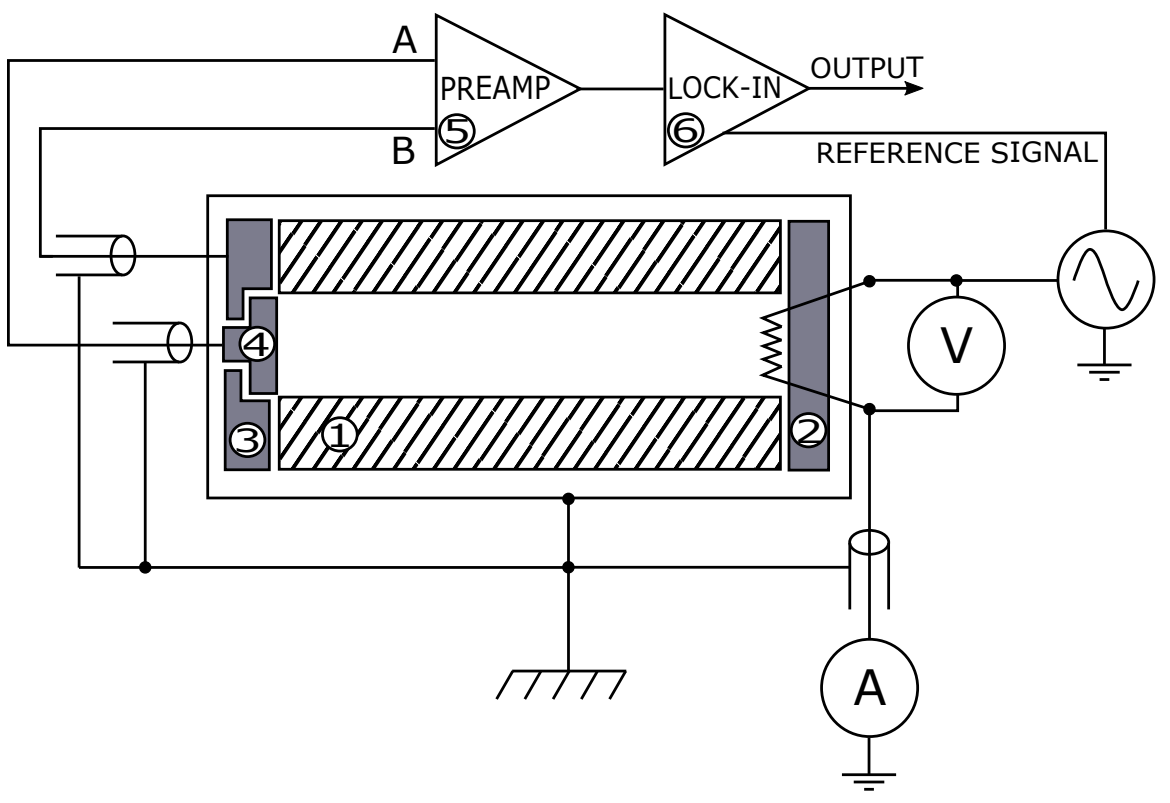

Figure 1: Electrical schematic of a typical resonator: (1) epoxy body of the resonator, (2) brass plate with heater, (3) brass mount with (4) brass electrode for detecting the electric response, (5) low-noise voltage preamplifier SR 560 from Stanford Research Systems, and (6) SR 830 lock-in amplifier.

sound can be calculated as:

$$
f_{r}=\frac{1}{4} \frac{n u_{2}}{L}
$$

where $u_{2}$ is value of the second sound velocity, $L$ is the actual length of the resonator and $n$ is the number of the half-wave lengths of the second sound signal for the observed resonance mode. The factor $1 / 4$ is caused by doubling the emission frequency of the heater and because the first resonance occurs when there is half of a second sound wavelength between the transmitter and receiver.

The actual length of the resonator was defined using a set of measurements of the second sound resonance. For this purpose, the resonator end opposite to the heater was covered by a polished brass disk with a Ge film thermometer glued to the center of the disk [14]. The low-inertia thermometer ensured the complete thermal tracking of the second sound waves. The thermometer was energized with a $1 \mu \mathrm{A} \mathrm{DC}$ current; to detect the signals, we used a SR830 dual phase lock-in amplifier. To excite the second sound wave, we used an Agilent 33250A function generator to drive the sinusoidal pattern of the heater.

The wave forms of the resonant modes are sinusoidal in space, the velocity fields have nodes at the ends of the resonators, and the temperature oscillations have antinodes coinciding with the velocity nodes. The observed second sound resonance peaks have Lorentzian shapes. Our resonator had a quality factor of approximately 60, and the actual length of the resonator $\mathrm{L}=24.3 \mathrm{~mm}$. The relatively low value of the resonator's actual length, as compared with its geometrical length, may be attributed to the heater thickness, which is slightly larger in the resonator. The non-flat form of the heater is 
presumably a reason for the low value of the quality factor.

\section{Electric response}

To study the electric response of He II in the resonator generated by a standing halfwave of second sound, the plate with the thermometer was replaced by a cylindrical electrode. The electrode, which had a thickness of $3 \mathrm{~mm}$ and a diameter of $7 \mathrm{~mm}$, was glued with Stycast in situ inside the brass mount.

We considered several measuring circuits depending on the nature of the phenomenon and the quality of the detected signal. Measurements of the electric potential between the plates fixed on both ends of the resonator would be a relatively reasonable way to study the bulk polarization of helium atoms. In this case, the electrode and the brass plate with the heater had a differential voltage connection (A-B) to the lock-in, whereby coaxial cable (A) was connected to the electrode and coaxial cable (B) connected to the plate on the opposite side of the resonator. The observed noise level in this case was approximately $10^{-5} \mathrm{~V}$, even for small drive amplitudes, whereas the amplitude of the required signal was supposed to be on the order of $10^{-7} \mathrm{~V}$. As a result, the high noise level made signal detection completely impossible. Although there was no electric connection between the heater and the plate, the proximity of the heater to the plate led to the appearance additional electromagnetic noise between the electrodes.

Another possible method is to measure the potential difference between the electrode and the ground. In this circuit, the noise of the signal was approximately one order of magnitude lower than in the previous case. It was possible to observe a resonance peak, but only at higher drive amplitudes $\left(\sim 10^{*} 10^{-3} \mathrm{~W} / \mathrm{cm}^{2}\right)$. We assume that the noise that arises from the coaxial cable's shield is the reason for the low resolution of the observed signal.

A significant enhancement of the signal-to-noise ratio was achieved when the electrode and its mount were connected to the lock-in differentially. Figure 1 shows a principal scheme of the resonator with the electrical connections that we used to study electrical activity in He II. Basically, the lock-in measures the voltage difference between the center conductor of coaxial cable (A) connected to the electrode and coaxial cable (B) connected to the electrode's mount. Noise pickup on the shield has no influence on the noise in the signal because the shields are ignored. The capacitance between the electrode and the mount was approximately $20 \mathrm{pF}$ (losses 0.025 ), and the total capacitance of the cables was $\sim 260 \mathrm{pF}$ (losses 0.02). In some experiments, a low-noise voltage preamplifier with differential voltage input (SR 560 from Stanford Research Systems) was added to the measuring loop. The use of a preamplifier with a bandwidth filter helped to reduce the interfering noise that appears due to mechanical vibration of the nanovolt level. This scheme allows for the measurement of charge density only in a thin layer of helium near the electrode. Thereby, it is difficult to determine the type of charge distribution that takes place in the resonator volume.

The electric response generated by the second sound wave was observed in the temperature range 1.71-2.04 K. An example of the electric response resonance is presented in Figure 2, The amplitude and shape of the observed resonance peaks were very sensitive to the temperature stabilization of the helium bath. The magnitude of the resonance dramatically decreases when the temperature fluctuation is on the order of $500 \mu \mathrm{K}$; thus, 


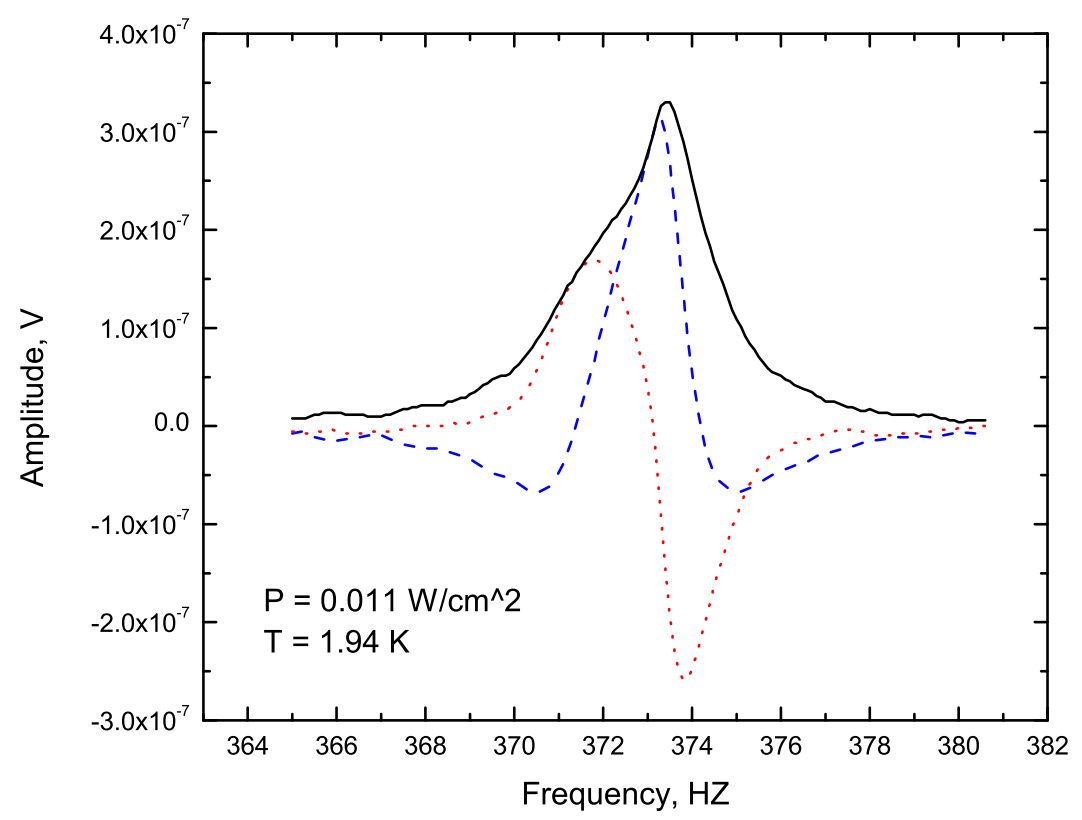

Figure 2: (Color on-line) The form of the second mode of the electric response signal plotted against frequency, showing the absorption (blue dashed curve) and dispersion (red dotted curve) data measured at $1.94 \mathrm{~K}$ with AC heat flux $0.011 \mathrm{~W} / \mathrm{cm}^{2}$. The black curve corresponds to the signal magnitude.

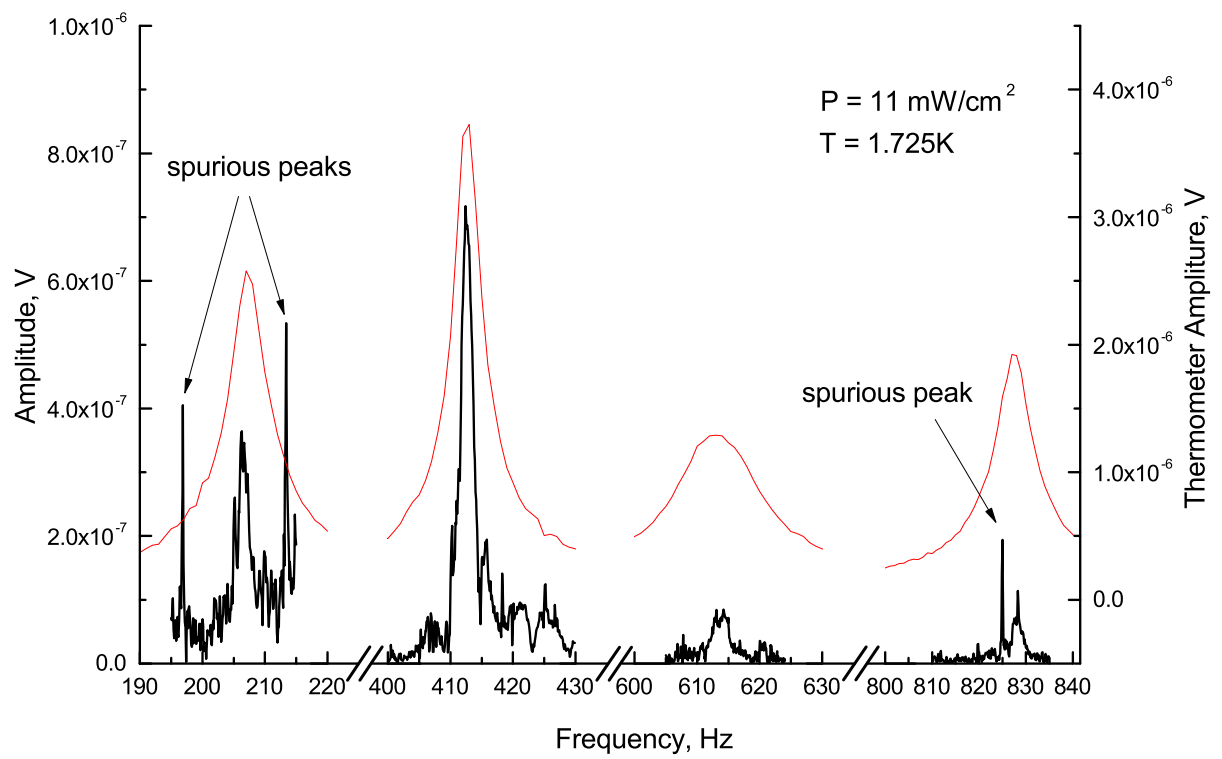

Figure 3: (Color on-line) The resonance peaks of the electric response of the first four harmonics (bottom black curves) measured at $1.73 \mathrm{~K}$. The top red curves represent the experimental data collected for the second sound resonances using the same harmonics. 


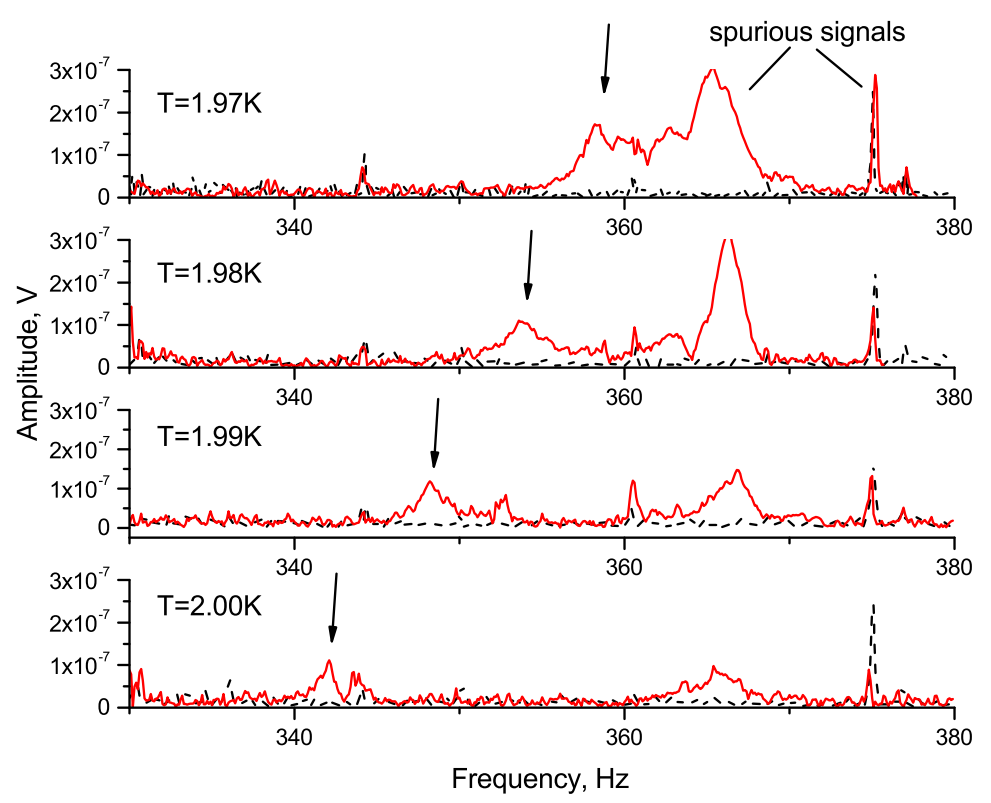

Figure 4: (Color on-line) Changing the resonance frequency of the standing wave by changing the bath temperature. The frequency range corresponds to the resonance frequency of the second harmonics in the temperature range $1.97-2 \mathrm{~K}$. The $\mathrm{AC}$ power heat flux was approximately $0.011 \mathrm{~W} / \mathrm{cm}^{2}$ at all temperatures. The black dashed curves correspond to the frequency sweet with zero excitation current applied to the heater. Black arrows indicate the frequency of electric induction resonance.

the signal was barely distinguishable from the background noise.

The signal of the electrical activity was observed using the first four harmonics (see Figure 3). In most cases, the highest peak amplitude was observed for the second harmonic, while for the third and fourth harmonics, the signal was almost lost in noise. The resonance frequencies of all four harmonics are in a good agreement with the resonance frequencies of the second sound that was independently measured in the same resonator.

It should be noted that a difference between the width of second sound resonance and the width of electric response was not observed in Ref. [3]. In our experiment, the width of second sound resonances was approximately two times higher than the width of electric response. Moreover, the values of width could change from measurement to measurement. As noted in Ref. [15], the reason for this low accuracy is low quality factor of the resonator.

In addition to the main peak that represented the electric response, we also detected a number of spurious signals. For example, there are two sharp peaks in Figure 3, which are located at the left and right sides of the first resonance mode. The magnitudes of these peaks are comparable or even higher than the required signal. We assume that mechanical vibrations from the pumping unit lead to a microphonic effect in the coaxial cables that appears as resonance peaks of a certain frequency.

For further study, we choose the second harmonic because of the higher amplitude of the signal and smaller number of spurious peaks compared with the first mode. Nevertheless, a frequency sweep in a range of interest chosen nearby the frequency of the second sound resonance revealed a number of peaks that might be misinterpreted as the 


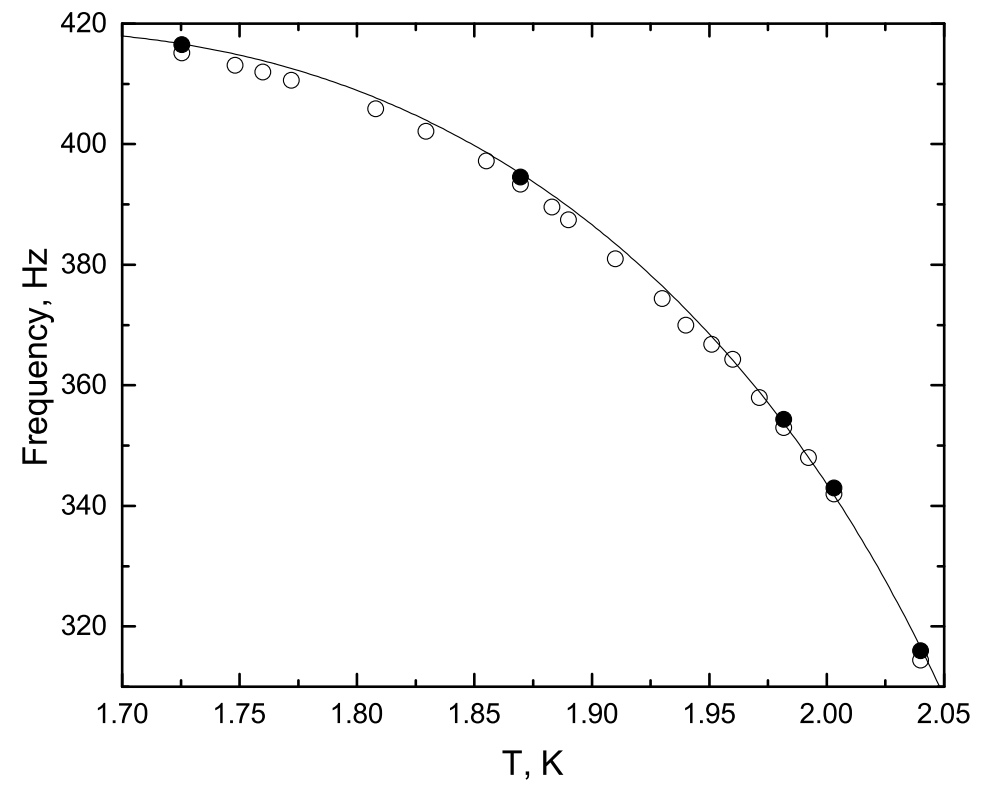

Figure 5: Temperature dependence of the resonance frequency of the electric response (open circles). The closed circles correspond to the resonance frequency the second sound. The solid line represents the resonance frequency of the second sound calculated for the resonator based on known data of the second sound velocity.

signal of electrical activity in He II (see the red curve in Figure 4, top graph). To exclude the possible influence of electric pickup on the signal, the frequency was swept with zero excitation current applied to the heater (see the black dashed curve in Figure 44). In this case, the resonance signal was not observed in the expected frequency range, but it was also observed that some spurious peaks disappeared from the frequency sweep.

Taking into account the difficulty in defining the required signal, the following measuring protocol was implemented. As was shown in Ref. 3, 44, the resonance frequency of the electric response is strongly dependent on the temperature. Performing measurements at several temperatures that were slightly different (about 10-30mK) from the specified temperature revealed a frequency shift for one of the peak signals. The shift of the resonance frequency was proportional to the change in the second sound velocity at the given temperature. Thus, this peak was defined as a resonance of the electric response. Figure 4 shows the frequency sweep for 4 different temperatures. The resonance that represents the electric response (marked by black arrows) shifts gradually as the temperature varies. There are a number of other peaks in this frequency range, but the resonance frequencies of these peaks is temperature independent. Therefore, we consider them to be spurious peaks. 


\section{Discussion}

The dependence of the resonance frequency for the second mode as a function of temperature is presented in Figure 5. These data can be compared with the value of the second sound resonance frequency calculated for our channel using Eq. 1. A small discrepancy between the calculated value of the resonance frequency and the value observed in the experiment can be explained by the slight differences in the geometry of the resonator. We calculated the resonator length based on the resonance frequency of the second sound observed in our resonator. The thermometer used in the second sound experiment had a thickness of approximately $1 \mathrm{~mm}$ and was slightly recessed into the cavity, which reduced its actual length. As a result the resonance frequency of the second sound is shifted toward higher frequencies with respect to that of the electric response. For example, decreasing the resonator length by $1 \mathrm{~mm}$ increases the resonance frequency by approximately $7 \mathrm{~Hz}$. In our experiment, the difference between the experimental and calculated values is about $4 \mathrm{~Hz}$, which is consistent with the difference in length between the two experiments.

Measurements of second sound resonance can be made simultaneously with a measurement of the electric response. In this case, the thermometer and electrode must be placed close to each other on one side of the resonator. However, the periodic oscillation of voltage on the thermometer due to the temperature oscillation in the second sound wave may induce some pickup signal on the electrode. To avoid potential sources of electric pickup, the measurements of electric response and second sound resonance were made in two different experiments.

Despite the low quality factor of the resonator in comparison with previous research, the absolute values of resonance amplitude observed in our experiment are higher than the one observed previously. The ratio $\Delta T / \Delta U$ is on the order of one hundred, while the value presented in the original article was two orders of magnitude higher. We believe that the discrepancy between the two experiments can be understood as follows. In both experiments, a potential difference, $\Delta U$, was measured on the electrode. The magnitude of $\Delta U$ can be determined as $\Delta U=\Delta Q / C_{i n}$, where $C_{i n}$ is the input capacitance. However, the parameters of the electrode, such as its capacitance and cross-section, were different in the two experiments. Therefore, the value of $\Delta U$ must be reconsidered in terms of the number of charges per unit area. In our resonator, this value is on the order of 1000 for the similar $\Delta T$ values reported in the original papers. The reports [3, 4] do not provide information about the capacitance or the surface area of the electrode, which makes it difficult to compare the results. It can be estimated that the discussed parameters of the electrode were somewhat smaller in the original experiment.

\section{Conclusions}

We have presented an experimental study of the electrical activity of superfluid helium that appears at the relative oscillatory motion of the normal fluid and superfluid components. The created apparatus allowed us to register an A.C. electric potential of approximately $5^{*} 10^{-7} \mathrm{~V}$. Our data give suggest the presence of an electric potential in superfluid helium excited by a second sound standing wave. For the first time, the electric response was observed for the first four harmonics with resonance frequencies that were similar to the frequency of second sound resonance. The resonance frequency 
of the electric response was compatible with the frequency of second sound resonance in the temperature range 1.72-2.04K. Despite the weak connection between the inner space of the resonator and the helium bath, the amplitude of the resonance peak was very sensitive to thermal fluctuation of the helium bath. We suggested a reliable method for the detection of the electric response that allows the required resonance to be distinguished from spurious signals. Our results are in qualitative agreement with the data published by previous researchers. Nevertheless, the amplitude of the resonance peak was higher despite the lower quality factor of the resonator. The reason for this discrepancy in the absolute values of the potential difference lies in the parameters of the electrode, such as the cross-section and capacitance. We believe that further investigation of this phenomenon could lead to a more complete understanding of the electric properties of superfluid helium.

Acknowledgements: The authors appreciate the technical assistance of F. Soukup and stimulating discussions with A. Rybalko, E. Rudavskii, L. Skrbek and V. Chagovets. This work is supported by the Czech Science Foundation (GACR) under Project No. 13-03806P.

\section{References}

[1] L. Tisza, Nature 141913 (1938)

[2] L. D. Landau, J. Phys. (USSR) 5, 71 (1941)

[3] A.S. Rybalko, Low Temp. Phys. 30, 994 (2004)

[4] A.S. Rybalko, et al. J. Low Temp. Phys. 148, 527 (2007)

[5] V. D. Natsik, Low Temp. Phys. 34, 493 (2008)

[6] A. M. Kosevich, Low Temp. Phys. 31, 37 (2005)

[7] L. A. Melnikovsky, J. Low Temp. Phys. 148, 559 (2007)

[8] M. D. Tomchenko, Phys. Rev. B 83, 094512 (2011)

[9] V.M. Loktev, M. D. Tomchenko, J. Phys. B At. Mol. Opt. Phys. 44, 035006 (2011)

[10] M. D. Tomchenko, J. Low Temp. Phys. 158, 854 (2010)

[11] S. I. Shevchenko, A. S. Rukin, Low Temp. Phys. 37, 884 (2011)

[12] E. A. Pashitskii, A. A. Gurin, J. Exp. Theor. Phys. 111975 (2011)

[13] In our experiment we use LakeShore coax cable with nominal capacity about 170 $\mathrm{pF} / \mathrm{m}$.

[14] V. F. Mitin, Advances in Cryogenic Engineering 43, (1982)

[15] K. N. Zinoveva, Sov. Phys. JETP 25, 2(8), 235 (1953) 